\title{
On the Alleged Extra-Structures of Quantum Mechanics
}

\author{
Davide Romano*
}

\begin{abstract}
I argue that a particle ontology naturally emerges from the basic dynamical equations of non-relativistic quantum mechanics, when the quantum continuity equation is realistically interpreted. This was recognized by J.J. Sakurai in his famous textbook "Modern Quantum Mechanics", and then dismissed on the basis of the Heisenberg position-momentum uncertainty principle. In this paper, I show that the reasons of this rejection are based on a misunderstanding of the physical import of the uncertainty principle. As a consequence, a particle ontology can be derived from the quantum formalism without the need of additional ad hoc assumptions, and therefore it cannot be regarded as "extra-structure".
\end{abstract}

\section{Contents}

1 Introduction

2 Derivation of the velocity law from the Schrödinger equation

3 The Statistical Character of the Uncertainty Principle 5

3.1 Historical and modern formulation of the uncertainty principle . . . . . . . 5

3.2 The uncertainty principle as a statistical principle . . . . . . . . . . . 6

3.3 On the impossible position-momentum measurements . . . . . . . . . . 8

3.4 Physicists look at the uncertainty principle . . . . . . . . . . . . . 9

3.4.1 Beyond the $\frac{\hbar}{2}$ limit: Ballentine . . . . . . . . . . . . . . 9

3.4.2 Statistical interpretation: Margenau, Ballentine, Bowman . . . . . . . 10

4 Philosophical discussion and conclusion $\quad 11$

4.1 A concrete example: relative phase and momentum distribution . . . . . . . . . 11

4.2 Statistical interpretation and individual description . . . . . . . . . . . . . 13

5 Conclusions $\quad 15$

${ }^{*}$ Centre of Philosophy, School of Arts and Humanities, University of Lisbon. Address: Alameda da Universidade, 1600-214, Lisbon, Portugal. Email: dromano@letras.ulisboa.pt 


\section{Introduction}

What is quantum mechanics about? Quantum systems are mathematically represented by the wave function, which evolves according to the Schrödinger equation and, when a measurement is performed, it collapses in one of the eigenstates of the measured observable (with statistical distribution given by the Born rule). So, a first answer seems to be: quantum mechanics is about the behavior of the wave function. In particular, the absolute square of the wave function $|\psi|^{2}$ is interpreted as the probability density to find the particle in a given region if a measurement of position is performed, and the corresponding integral as the probability to find the particle on that region. However, this answer suddenly raises (at least) two problems:

1. the physical meaning is linked to the absolute square of the wave function, not to the wave function per se. This leaves the question about the nature of the wave function unanswered (and maybe unanswerable) in the standard context;

2. there is a perfect symmetry between the wave function represented in position and momentum space: which one of the two should be considered as the correct candidate for the ontology of the theory? And why?

In the standard interpretation, the correct answer of this second point is neither of them: the fundamental entity of quantum mechanics, mathematically speaking, is not the wave function but the state vector. Wave functions in position and momentum spaces are, indeed, different mathematical representations of the same state vector. Insofar there is perfect symmetry between wave functions expressed in different bases, the state vector should be regarded as a more fundamental entity of the theory. We are thus eventually led to the conclusion that the fundamental entity of standard quantum mechanics is the state vector of a system: a ray (given the equivalence class for overall phase multiplication) mathematically defined in the Hilbert space $\mathcal{H}$ of the system. But then the initial question -what is quantum mechanics about?- far from being solved, have become even more tricky: what does a state vector actually represent in the physical world? What is the ontological connection between state vectors in Hilbert space and physical systems in three-dimensional space described by quantum mechanics, such as electrons, atoms, molecules, and eventually chairs, cats and human beings? ${ }^{1}$

The answer is rather simple though: the state vector does not have a physical meaning per se in quantum mechanics. The theory, according to the standard/textbook interpretation, is neither about the wave function nor the state vector: it is about observables - mathematically described by Hermitian operators - and the (real-valued) spectrum of possible eigenvalues. The observables' eigenvalues eventually form the direct link between the formalism (state vector, Schrödinger equation, collapse postulate) and the physical content of the theory (measurement outcomes in the laboratory, collected in empirical distributions). Therefore, what standard quantum mechanics really does is not to describe the evolution of systems (through the wave function or the state vector) but uniquely to assign definite values (the eigenvalues) to every physical property (observable) we can practically measure in a laboratory. That is, it correctly describes the statistics of measurement outcomes without providing a description of the quantum objects and the physical processes involved at the microscopic regime and responsible for the concrete realization of the macroscopic results. ${ }^{2}$

Contrary to this standard view, I will argue that quantum mechanics can be naturally interpreted as a theory of particles in motion. With this, I do not intend that the ontology of

\footnotetext{
${ }^{1}$ Cats, chairs and human beings, even if classical objects, are fundamentally quantum in nature, for they are composed of molecules and atoms at the basic level. In fact, classical mechanics is basically quantum mechanics in a specific (macroscopic and decoherence) regime (see e.g. (Holland 1993, Ch. 6), Schlosshauer (2007)).

${ }^{2}$ The measurement and registration of a certain eigenvalue is always mediated by a macroscopic device.
} 
quantum mechanics is just about particles, or even primarily about particles (the nature of the wave function is put aside), but that the very formalism of quantum mechanics contains structures that are not usually recognized in the standard interpretation, on the contrary they are usually (and misleadingly) refereed to as "extra-structures". These are continuous position and velocity variables - particles in motion- that can be naturally derived from the quantum continuity equation when this is realistically interpreted. This was recognized by Sakurai (1994), but then dismissed on the basis of the position-momentum uncertainty principle. However, when the uncertainty principle is carefully analyzed, it becomes apparent that it does not prevent a simultaneous assignment of position and momentum values on individual systems, i.e. it does not prevent the existence of particles in motion. The idea is that, if particles' trajectories can be mathematically derived from (and not added to) to the basic equations of the theory, and the physical interpretation of such trajectories is not forbidden but, on the contrary, even suggested by a realistic interpretation of the theory, then it is hard to regard such a particle ontology as extra-structure.

The structure of the paper is the following: in Section 2, I recall Sakurai's derivation of the velocity formula from the Schrödinger equation; in Section 3, I analyze the physical import of the Heisenberg uncertainty principle and show that, contrary to Sakurai's claim, it is not in conflict with the existence of particles' trajectories; in Section 4.1, I describe a concrete example (involving relative phase and momentum distribution) that can be intuitively explained (for simple cases, at least) by taking into account the actual particles' velocity. I conclude (Section 4.2) with a philosophical discussion on the results of the paper and the need for an individual description of systems that goes beyond the statistical character of standard quantum mechanics.

\section{Derivation of the velocity law from the Schrödinger equation}

In his textbook Modern Quantum Mechanics, (Sakurai 1994, pp. 101-102) derives the particle velocity formula from the standard quantum formalism. In this section, I shall summarize the main steps of Sakurai's original derivation, which will serve as a basis to assess and defend the main thesis of the paper. In fact, such derivation is interesting for (i) it shows the precise connection between the standard formalism and the derivation of moving particles and (ii) it indicates that the velocity variable should not be considered as added but derived within the formalism itself. As a first step, we consider the Schrödinger equation for a spinless 1-particle system: ${ }^{3}$

$$
i \hbar \frac{\partial \psi(x, t)}{\partial t}=\hat{H} \psi(x, t)=-\left(\frac{\hbar^{2}}{2 m}\right) \nabla^{2} \psi(x, t)+V \psi(x, t)
$$

where $i$ is the imaginary unit, $\hbar$ the reduced Plank constant and $\hat{H}$ the Hamiltonian operator. From (1) we can derive the quantum continuity equation for the probability density $\rho(x, t)=$ $|\psi(x, t)|^{2}$ :

$$
\frac{\partial|\psi(x, t)|^{2}}{\partial t}+\nabla \cdot J(x, t)=0
$$

where $J$ is the quantum flux, also known as the quantum probability current. Written explicitly, $J$ takes the following form:

$$
J(x, t)=\frac{i \hbar}{2 m}\left(\psi^{*} \nabla \psi-\psi \nabla \psi^{*}\right)=\frac{\hbar}{m} \operatorname{Im}\left(\psi^{*} \nabla \psi\right) .
$$

\footnotetext{
${ }^{3}$ While we consider here, for simplicity, a 1-particle system, the present derivation can be straightforwardly generalized to $N$-particle systems.
} 
As a second step, we decompose the wave function in polar form:

$$
\psi(x, t)=R(x, t) e^{\frac{i}{\hbar} S(x, t)}
$$

where $R(x, t)$ and $S(x, t)$ are real-valued functions representing, respectively, the amplitude and phase of the wave function. We can now express the probability density in terms of the amplitude as $\rho(x, t)=R^{2}(x, t)$ and make the following substitution:

$$
\psi^{*} \nabla \psi=R \nabla R+\frac{i}{\hbar} R^{2} \nabla S
$$

The quantum probability current thus becomes:

$$
J=\frac{\hbar}{m} \operatorname{Im}\left(R \nabla R+\frac{i}{\hbar} R^{2} \nabla S\right)=\frac{\hbar}{m} \frac{R^{2} \nabla S}{\hbar}=\frac{R^{2} \nabla S}{m},
$$

which we can rewrite (using the relation $\rho=R^{2}$ ) as follows:

$$
J=\frac{\rho \nabla S}{m}=\rho v
$$

having defined the velocity term as:

$$
v=\frac{\nabla S}{m}
$$

which corresponds to the velocity of the particles in the de Broglie-Bohm theory. ${ }^{4}$ Eq. (4) is an important result: the quantum flux can be decomposed into a particle density term and a particle velocity term, analogously to the classical flux in fluid dynamics. From this general consideration, it follows the identification of $v=\frac{\nabla S}{m}$ as a velocity formula and the possibility to interpret the quantum flux in realistic terms:

We now see that there is more to the wave function than the fact that $|\psi|^{2}$ is the probability density; the gradient of the phase $S$ contains a vital piece of information. From (4) [equation number adapted] we see that the spatial variation of the phase of the wave function characterizes the probability flux; the stronger the phase variation, the more intense the flux (Sakurai 1994, 104).

One argument in support of a realistic interpretation of the quantum flux (and the quantum continuity equation, more generally) is given by the fact that, since in standard quantum mechanics is not the wave function per se but its absolute square to have a physical meaning (probability density), we expect the dynamical equation associated to this quantity to have a precise physical meaning as well. Such a dynamical equation -the time evolution of the absolute square of the wave function- is the quantum continuity equation and, therefore, we expect this equation to have a strong physical meaning. A second argument is provided by the strict analogy between the quantum probability current and the classical hydrodynamic current:

$$
J=\rho v \Longleftrightarrow J_{c l}=\rho_{c l} v,
$$

\footnotetext{
${ }^{4}$ Historically, de Broglie wrote his particle dynamics in 1926/1927 before quantum mechanics was even completed (the original idea was to unify two principles of analytical mechanics: the principle of Maupertuis and the principle of Fermat, so deriving a new particle-wave dynamics), and Bohm (1952) derived the velocity formula from the quantum Hamilton-Jacobi equation. In both cases, the velocity was not added to the Schrödinger equation but mathematically derived (Bohm) or independently formulated as the new dynamics for quantum systems (de Broglie).
} 
which reduces the quantum continuity equation to the classical continuity equation:

$$
\frac{\partial R^{2}}{\partial t}+\nabla \cdot\left(R^{2} \frac{\nabla S}{m}\right)=0 \longrightarrow \frac{\partial \rho}{\partial t}+\nabla \cdot(\rho v)=0 .
$$

That is: exactly as in classical fluid dynamics, we can interpret the quantum flux as a real flux of particles in motion $\psi^{2}$-distributed and moving with velocity $v=\nabla S / \mathrm{m}$. In this respect, we can say that (i) particle positions and velocities are not added to the standard formalism but derived from it and (ii) a particle ontology naturally emerges from standard quantum mechanics once the quantum continuity equation is interpreted in realistic terms.

Nevertheless, Sakurai did not interpret the term $v=\nabla S / m$ as a real velocity, since -he arguesthis would imply a violation of the position-momentum uncertainty principle:

\begin{abstract}
However, we would like to caution the reader against a too literal interpretation of $J$ as $\rho$ times the velocity defined at every point in space, because a simultaneous precision measurement of position and velocity would necessarily violate the uncertainty principle (Sakurai 1994, 102-103).
\end{abstract}

Sakurai's assumption is that the uncertainty principle refers to simultaneous position and momentum measurements on individual systems. However, a careful reading of this principle suggests to interpret it as a statistical principle, i.e. a mathematical relation that applies not to individual systems but to ensembles of systems (sequences of individual systems, all represented by the same initial state). When statistically interpreted, the uncertainty principle does not prevent to assign simultaneous well-defined values of position and momentum to individual systems, i.e. it does not prevent the description of particle trajectories for quantum systems.

\title{
3 The Statistical Character of the Uncertainty Principle
}

\subsection{Historical and modern formulation of the uncertainty principle}

The position-momentum uncertainty principle, expressed by the following inequality:

$$
\Delta Q \Delta P \geq \frac{\hbar}{2}
$$

where $\hbar$ is the reduced Planck constant and $\Delta Q$ and $\Delta P$ are the standard deviations of, respectively, the position and momentum operator, has been originally derived in this form by Kennard (1927). This is the standard formula of the uncertainty principle that we find in contemporary textbooks on quantum mechanics. Nonetheless, (7) is not the way in which Heisenberg originally formulated the principle. In fact, Heisenberg expressed the uncertainty relation as an epistemic principle, that is, a principle coming from the experimental inaccuracy of position and momentum measurements performed on individual systems. Heisenberg did not give a rigorous proof for this relation: the arguments in support of the principle were, on the contrary, pretty qualitative, as noted e.g. by Hilgevoord and Uffink (2016). We can mathematically express the original Heisenberg uncertainty principle as follows ${ }^{5}$ :

$$
\delta q \delta p \geq h
$$

where $h$ is the Planck's constant. In this case, $q$ and $p$ are not the position and momentum operators, but the position and momentum eigenvalues (i.e. the values of the position and

\footnotetext{
${ }^{5}$ Hilgevoord and Uffink (2016) provide a detailed reconstruction of Heisenberg's arguments in support of this form for the uncertainty principle.
} 
momentum of the system that have been measured in the experiment) and $\delta$ is the level of inaccuracy or experimental error associated to the measurement.

From these considerations, it is possible to contrast the original Heisenberg's formulation of the uncertainty principle, characterized as an empirical relation concerning the accuracy of measurements, with its modern standard formulation (7), which is a theorem of quantum mechanics, since it can be mathematically derived within the formalism of the theory. Specifically, relation (7) can be obtained as a particular case of the general inequality [Robertson (1929)]:

$$
\Delta A \Delta B \geq \frac{1}{2}|\langle[A, B]\rangle|
$$

where $[A, B] \equiv A B-B A$ is the commutator of the operators $A$ and $B$ and $|\cdot|$ the absolute value, through the substitution of the general observables $A$ and $B$ with the position and momentum observables $Q$ and $P$. In the next subsections (3.2-3.4), I will analyze the philosophical and physical meaning of the Heisenberg uncertainty relation as expressed by the formula (7), since (i) this is the textbook modern formulation of the principle and (ii) this is the formulation of the pricniple invoked by Sakurai to dismiss the possibility that eq. (5) represents a real particle velocity. From the analysis, however, it will be clear that there is no conflict between the position-momentum uncertainty principle and a simultaneous assignment of well-defined values of position and velocity (i.e. trajectories) for individual systems.

\subsection{The uncertainty principle as a statistical principle}

The textbook formulation of the Heisenberg uncertainty principle is expressed by relation (7), where $Q$ and $P$ represent, respectively, the position and momentum operators, and $\Delta$ is the quantum uncertainty, i.e. the standard deviation of the associated operator. In order to understand the physical import of this relation, it may be useful to analyze what the uncertainties $\Delta Q$ and $\Delta P$ primarily refer about. In fact, claims that the uncertainty principle prevents systems to have simultaneous well-defined values of position and momentum usually come from a misleading interpretation of those quantities. Let us take a closer look at the description of a measurement in quantum mechanics: this will allow to clarify the relation between (single) measurement outcomes, empirical distributions and the quantum uncertainties $\Delta Q$ and $\Delta P$.

According to standard quantum mechanics, any measurable property of a system (position, momentum, energy, etc.) is called "observable" and is mathematically represented by an Hermitian operator. Operators are defined by an eigenvalue equation that describes how they act on the system's state. Suppose, for example, that we want to measure a certain property, mathematically represented by the operator $A$, of a system with initial state $\psi$. We can then define an eigenvalue equation of the type:

$$
A\left|a_{i}\right\rangle=a_{i}\left|a_{i}\right\rangle
$$

where $\left|a_{i}\right\rangle$ are the eigenstates of $\mathrm{A}$ and $a_{i}$ the associated eigenvalues. Since Hermitian operators have a real spectrum, the eigenvalues are real numbers. However, when we measure an observable A on a given system, the initial state of the system is usually not an eigenstate of A. Nevertheless, it is always possible to represent the state as a coherent superposition of eigenstates of $\mathrm{A}:^{6}$

$$
|\psi\rangle=\sum_{i} c_{i}\left|a_{i}\right\rangle
$$

The result of a single measurement, i.e. the unique outcome recorded by the measuring device in the laboratory after its interaction with the quantum system, can thus only be, according

\footnotetext{
${ }^{6}$ For simplicity, we suppose that the observable $A$ has a discrete spectrum of eigenvalues. For observables having a continuous spectrum of eigenvalues, such as position, the basis expansion involves the Dirac's $\delta$ function. However, this mathematical subtlety does not affect the argument we are presenting here.
} 
to the formalism of the theory, one of the possible eigenvalues $a_{i}$, say $a_{m}$, associated to the eigenstate $\left|a_{m}\right\rangle$ of the observable $A$ :

$$
\sum_{i} c_{i}\left|a_{i}\right\rangle \stackrel{H_{\text {int }}}{\longrightarrow} a_{m}\left|a_{m}\right\rangle
$$

where $H_{\text {int }}$ represents the measurement interaction. The probability to obtain the eigenvalue $a_{m}$ in a measurement is given by the Born rule: $P\left(a_{m}\right)=\left|c_{m}\right|^{2}=\left|\left\langle a_{m} \mid \psi\right\rangle\right|^{2}$. The same procedure applies to measurements of position and momentum: when we measure the position of a system we always obtain a single well-defined value, i.e. one of the eigenvalues of the position operators. The same for the momentum operator. But then -we may ask- where do the uncertainties $\Delta Q$ and $\Delta P$ come from? Short answer is: they are, mathematically speaking, the standard deviations of the probability distributions of the position and momentum operators. As such, they emerge from and can be computed after a (reasonably long) sequence of single measurements on identically prepared systems. We recall indeed that the standard deviations of the position and momentum operators are defined by the formulae:

$$
\begin{aligned}
& \Delta Q=\sqrt{\left\langle Q^{2}\right\rangle-\langle Q\rangle^{2}}, \\
& \Delta P=\sqrt{\left\langle P^{2}\right\rangle-\langle P\rangle^{2}} .
\end{aligned}
$$

where $\langle Q(P)\rangle$ is the mean value of the position (momentum) operator:

$$
\langle Q\rangle=\int|\psi(x)|^{2} x d x
$$

The mean value, or average value, of an operator is a statistical quantity, for it can be computed only after a sequence of measurements on identically prepared systems. This can be clearly seen from the standard definition of the mean value in terms of the observable's eigenstates (this applies to every observable with a discrete spectrum of eigenvalues):

$$
\langle A\rangle=\sum_{i} a_{i}\left|\left\langle a_{i} \mid \Psi\right\rangle\right|^{2}
$$

In order to compute (meaningfully) the mean value $\langle A\rangle$, one needs to compute a sum over all eigenvalues of the observable $\mathrm{A}$, each averaged with the associated probability given by the Born rule. This is a theoretical computation of the mean value, but still, every (meaningful) empirical computation in a laboratory should refer to (13) as the theoretical measure, trying to approximate it as well as possible in the long run, i.e. after a long sequence of measurements performed on identically prepared systems. In other words: the computation of the mean value of an operator in laboratory can only be retrieved after a sequence of many measurements of the same observable performed on copies of identically prepared systems (i.e., systems with the same initial state). The standard deviation of a certain operator, as expressed by the formulas (10) and (11), is a quantity mathematically derived from the mean value of that operator. Therefore, it is (exactly as the mean value) a statistical quantity, which cannot refer or be associated to single measurements. Both the mean value and the standard deviation are therefore correctly related to the probability distributions of eigenvalues that are obtained after many measurements of the same observable on the system. Consequently, they are statistical quantities that are computed from empirical distributions of actual values (eigenvalues) obtained as measurement outcomes in the laboratory. From these considerations, it is clear that the Heisenberg uncertainty principle does not indicate a theoretical limitation on the precision of simultaneous measurements of position and momentum, but expresses a statistical relation: the 
standard deviation of the joint probability distributions of position and momentum operators cannot be arbitrarily small, but has a lower bound of $\hbar / 2$.

\subsection{On the impossible position-momentum measurements}

There is a further reason why the uncertainty principle does not refer to simultaneous measurements of position and momentum on individual systems: such measurements cannot practically be realized or performed in quantum mechanics. The formalism of quantum theory, indeed, forbids simultaneous measurements of different observables, unless they have a common basis of eigenstates (this condition is realized when the system state vector can be decomposed in states that are eigenstates of both observables). However, position and momentum are not observables of this sort. Instead, they are non-commuting, or "incompatible", observables:

$$
[\hat{x}, \hat{p}]=i \hbar .
$$

i.e., they do not have a common basis of eigenstates. Therefore, according to standard quantum mechanics, a simultaneous measurement of the position and momentum observables on a single system cannot be performed. Suppose, for example, that we want to perform a simultaneous measurement of two different generic observables $A$ and $B$. The result of the measurement interaction between the two observables and the system should be mathematically expressed by a common eigenbasis of the two measured observables, i.e. by a set of common kets that are eigenstates of both $A$ and $B$ :

$$
(A, B)|\psi\rangle=\sum_{j} c_{j}\left|k_{j}\right\rangle
$$

where $(A, B)$ denotes a simultaneous measurement of the observables $A$ and $B$, i.e. a physical interaction between the system (mathematically represented by $|\psi\rangle$ ) and a measurement device which is able to measure, at the same time, the eigenvalues of $A$ and $B,{ }^{7}$ and $\left|k_{j}\right\rangle$ represents a common basis of eigenstates of $A$ and $B$. More precisely, a basis such that:

$$
\begin{aligned}
& A \psi=\sum_{j} c_{j}\left|k_{j}^{a}\right\rangle \\
& B \psi=\sum_{j} c_{j}\left|k_{j}^{b}\right\rangle
\end{aligned}
$$

where $\left|k_{j}^{a}\right\rangle$ and $\left|k_{j}^{b}\right\rangle$ are the eigenstates of both observables $A$ and $B$ to which are associated, respectively, the eigenvalues $k_{a}$ and $k_{b}{ }^{8}$

We are now in the position to evaluate why a simultaneous measurement of position and momentum on an individual system cannot be performed. According to the formalism introduced above, a measurement of this kind is mathematically expressed as follows:

$$
(A, B)|\psi\rangle=\sum_{j} c_{j}\left|k_{j}^{a b}\right\rangle \stackrel{H_{i n t}}{\longrightarrow} k_{m}^{a}\left|k_{m}^{a}\right\rangle, k_{n}^{b}\left|k_{n}^{b}\right\rangle .
$$

The measurement interaction (14) would simultaneously provide two results: one single welldefined eigenvalue $k_{m}^{a}$ of the observable $A$, with probability $P\left(k_{m}^{a}\right)=\left|c_{m}\right|^{2}=\left|\left\langle k_{m}^{a} \mid \psi\right\rangle\right|^{2}$ and one

\footnotetext{
${ }^{7}$ Note that the expression $(A, B)|\psi\rangle$, indicating a simultaneous measurement of the observables $A$ and $B$, differs from $(A B)|\psi\rangle$, which usually denotes an experiment in which we perform a single measurement of the observable $B$ and, then, after a discrete amount of time, a second measurement of the observable $A$.

${ }^{8} \mathrm{I}$ assume, for simplicity, that the expansion coefficients $c_{j}$ are the same in the two cases. This is an ideal assumption: in general, they will be different for the two operators, provided that the eigenstates are the same.
} 
single well-defined eigenvalue $k_{n}^{b}$ of the observable $B$ with probability $P\left(k_{n}^{b}\right)=\left|c_{n}\right|^{2}=\left|\left\langle k_{n}^{b} \mid \psi\right\rangle\right|^{2}$. This interaction, however, can be concretely written down-and the corresponding experimental procedure concretely be realized in a laboratory-only if the observables $A$ and $B$ have a common basis of eigenstates $\left(\left|k_{j}\right\rangle\right)$, i.e., only if $\mathrm{A}$ and $\mathrm{B}$ commute: $[A, B]=A B-B A=0$. But, as noted before, position and momentum are non-commuting operators, as they do not have a common basis of eigenstates. Consequently, a simultaneous measurement of position and momentum cannot be performed, i.e. it cannot be realized an experiment which provides, as measurement result, two eigenvalues, one for the position and one for the momentum operator, simultaneously. This is certainly a cogent reason why the uncertainty principle does not concern simultaneous measurements of position and momentum-otherwise, that would be a principle about something that is not even allowed by the formalism of quantum mechanics.

We can finally summarize our analysis of the position-momentum uncertainty principle in the two points below:

1. The uncertainty principle is best regarded as a statistical principle that applies to ensembles (sequences of identically prepared) of systems and not to individual systems. In particular, it puts a lower bound equal to $\hbar / 2$ on the product of the standard deviations of the position and momentum probability distributions.

2. The uncertainty principle does not concern simultaneous measurements of position and momentum performed on individual systems, since this kind of measurement procedure is forbidden for non-commuting observables, such as position and momentum, by the basic formalism of quantum mechanics.

\subsection{Physicists look at the uncertainty principle}

The conclusion that the position-momentum uncertainty principle is best regarded as a statistical principle is however not an original thesis. This interpretation has been defended indeed by many physicists in the last decades, from 1960/'70 (e.g., Margenau, Ballentine) to more recent times (e.g., Bowman). In this subsection, I shall report some of the original arguments of these authors in favor of the statistical interpretation of the uncertainty principle. The scope is twofold: on the one hand, to give credit to those who have originally argued in favor of this position and, on the other hand, to discuss further examples and arguments in support of it.

\subsubsection{Beyond the $\frac{\hbar}{2}$ limit: Ballentine}

An interesting remark, originally made by Jauch (1993) and reported in Ballentine (1998), comes from cyistallography: Jauch describes an experimental situation in which the position and momentum of atoms in a crystal can be known with a precision greater than the limit imposed by the uncertainty principle $\hbar / 2$ :

The rms [root-mean-square] atomic momentum fluctuation, $\Delta P$, is directly obtained from the temperature of the crystal, and hence (7) [equation number adapted] gives a lower bound to $\Delta Q$, the rms vibration amplitude of an atom. The value of $\Delta Q$ can be measured by means of neutron diffraction, and at low temperatures it is only slightly above its quantum lower bound, $\hbar / 2 \Delta P$. Jauch stresses that it is only the rms ensemble fluctuations that are limited by (7). The position coordinates of the atomic cell can be determined with a precision that is two orders of magnitude smaller than the quantum limit on $\Delta Q$. (Ballentine 1998, p. 227, emphasis added) 
However, this is not a plain violation of the uncertainty principle, but an experimental indication that the latter does not prevent a simultaneous measurement of position and momentum on individual systems with a precision greater than $\hbar / 2,{ }^{9}$ and, eventually, with arbitrary precision (the latter depending only on the accuracy of the experimental set-up and the level of experimental error on individual measurements). ${ }^{10}$

\subsubsection{Statistical interpretation: Margenau, Ballentine, Bowman}

Historically, the statistical interpretation of the uncertainty principle has been originally defended by Margenau. She remarks indeed that the position and momentum "uncertainties" refer to the standard deviations of the measured observables and, as such, they are meaningfully defined only as statistical quantities:

Above all we note that $\Delta P$ or $\Delta Q$, like every statistical quantity, sustains no reference to a single observation: an assignment of $\Delta Q$ to one measurement is as empty as an attribution of temperature to one molecule. (Margenau 1963, p. 471)

Some years later Ballentine expressed a similar position, interpreting the quantum uncertainties $\Delta Q$ and $\Delta P$ as the probability distributions associated to, respectively, the position and momentum operators:

The results (9) and (7) [number of equations adapted] assert that for any particular state (i.e., state preparation) the product of the widths of the distributions of $A$ measurements and of $B$ measurements may not be less than some lower limit. A term such as the statistical dispersion principle would really be more appropriate for these results than the traditional name, uncertainty principle.

(Ballentine 1970, p. 364)

More recently, the statistical interpretation of the uncertainty principle has been defended by Bowman in his book Essential Quantum Mechanics:

[...] in the statistical interpretation, probabilities refer to ensembles, so there is no reason to interpret uncertainties as necessarily applying to simultaneous measurements of both $x$ and $p_{x}$ on a single system. We interpret $\Delta x$ and $\Delta p_{x}$ as the spreads in $x$ and $p_{x}$, respectively, that appear in making many separate measurements on identically prepared particles -one measurement on either $x$ or $p_{x}$, not both, per system. It may seem puzzling, but even in the simultaneous measurement approach, probabilities are always manifest in a series of measurements, never in a single measurement. (Bowman 2008, p. 91, original emphasis)

Interestingly, Bowman reduces the position-momentum uncertainty principle to the superposition principle; in particular, to the possibility we have in quantum mechanics to represent a certain quantum state in different bases (in the present case, the position and the momentum bases):

\footnotetext{
${ }^{9}$ The position and momentum eigenvalues of the atoms in the crystal are inferred from the total mean thermal energy of the crystal. The experimental accuracy goes beyond the uncertainty principle for temperatures lower than $T=15 K$.

${ }^{10}$ Ballentine remarks that the standard deviations $\Delta Q$ and $\Delta P$ cannot be properly built at all unless the level of experimental error on individual measurements $\delta Q$ and $\delta P$ be much smaller than the standard deviations.
} 
In a sense, however, they [the uncertainty relations] are simply a consequence of the superposition principle-the fact that we may expand a state in the eigenstates of different Hermitian operators, and from these expansions obtain the relevant probabilities. Different expansions yield different probability distributions, yet they cannot be independent, since all are extracted from the same state. The uncertainty relations quantify this fact. (Bowman 2008, p. 92)

On a careful analysis, the uncertainty principle is thus best regarded as a statistical principle. For this reason, contrary to Sakurai's claim, it cannot prevent the assignment of simultaneous well-defined values of position and momentum to individual systems. That is: it cannot prevent to regard the velocity term derived from the continuity equation as a proper velocity for the particles. The quantum continuity equation may then be interpreted as a flux of particles $|\psi|^{2}$-distributed and in motion with velocity $v=\frac{\nabla S}{m}$. Exact position and velocity variables are therefore not artificially added to the formalism of quantum mechanics: they can be mathematically derived from the basic equations of the theory. Finally, if the quantum continuity equation is realistically interpreted, these variables tend to assume an ontological status, describing a real quantum dynamics, i.e. a quantum theory of particles in motion. In the next section, I will expand on this important point.

\section{Philosophical discussion and conclusion}

\subsection{A concrete example: relative phase and momentum distribution}

Considering quantum mechanics as a theory of particles in motion is not just a theoretical exercise. It can also draw new light on some parts of the formalism that remain unexplained in the standard context. In this section, I illustrate how a particle ontology may provide an intuitive explanation of a specific result of quantum mechanics: the variation of the momentum probability distribution if the wave function is multiplied by a relative phase.

In quantum mechanics, if we multiply a (position-basis) wave function by a relative phase - a term that multiplies each position eigenstate for a different number - the position probability distribution remains unchanged, while the momentum probability distribution will be altered.

Consider a system described by the wave function ( $\operatorname{setting} \hbar=1$ ):

$$
\psi(x, t)=R(x, t) e^{i S(x, t)},
$$

we multiply it by the relative phase $e^{i X(x)}$ obtaining:

$$
e^{i X(x)} \psi(x, t)=e^{i X(x)} R(x, t) e^{i S(x, t)} .
$$

According to the standard formalism of quantum theory, the position probability distribution remains unchanged after the action of the relative phase:

$$
\left|R(x, t) e^{i X(x)} e^{i S(x, t)}\right|^{2}=|R(x, t)|^{2}=|\psi(x, t)|^{2}
$$

while the momentum probability distribution, on the contrary, will change. In order to show this fact, we transform the wave function from the position to the momentum basis:

$$
\phi(p, t)=N \int \psi(x, t) e^{-i p x} d x
$$

and multiply the momentum-basis wave function $\phi(p, t)$ by the relative phase $e^{i X(x)}$. We obtain: 


$$
e^{i X(x)} \phi(p, t)=N \int e^{i X(x)} \psi(x, t) e^{-i p x} d x .
$$

As we can see from the equations above, the two wave functions (15) and (16) have different momentum probability distributions. In the standard context of quantum mechanics, there is no physical explanation of this fact: it just follows from the basic rules of quantum mechanics. However, an intuitive explanation (if only for simple cases, as I will stress below) can be given if we take quantum theory to really describe particles in motion. This follows considering the mathematical relation between the term corresponding to the relative phase and the actual velocity of the particles.

In fact, the velocity of the particles as derived from the continuity equation is:

$$
v=\frac{\nabla S(x, t)}{m}
$$

and the multiplication by a relative phase is nothing but a change of the original phase $S(x, t)$ :

$$
e^{i X(x)} R(x, t) e^{i S(x, t)}=R(x, t) e^{i(X(x)+S(x, t))}=R(x, t) e^{i S^{\prime}(x, t)},
$$

which simply generates the new actual velocity:

$$
v^{\prime}=\frac{\nabla S^{\prime}(x, t)}{m}
$$

That is: when we multiply the wave function by a relative phase, the original phase of the wave function will chance and, since the velocity is determined by the gradient of the phase, also the actual velocity of the particles will change. The new velocity, i.e. real particles moving faster or slower with respect to the initial state, will eventually determine a new momentum probability distribution.

However, an important remark is in order. The momentum eigenvalue $p_{0}$ (eigenvalue of the momentum operator $\hat{P}$ ), defined as:

$$
\hat{P} \psi=-i \hbar \nabla \psi=p_{0} \psi
$$

and the real momentum of the particle $p_{r}$ :

$$
\nabla S=m v=p_{r}
$$

are conceptually distinct, and they do not always converge to the same value. In general, in fact:

$$
p_{0} \neq p_{r}
$$

For simple cases, however, they do converge, and the two (a priori distinct) entities take the same value. For example, consider a plane wave with momentum $p$ :

$$
\psi(x, t)=e^{\frac{i}{\hbar} p x}
$$

In this case, the momentum eigenvalue is $p_{0}=p$ :

$$
\hat{P} \psi(x, t)=-i \hbar \nabla \psi(x, t)=p \psi(x, t)
$$

which coincides with the real momentum $p_{r}$ of the particle: 


$$
\nabla S=\nabla(p x)=p=m v=p_{r}
$$

Nevertheless, it is worth noting that not always quantities related to the momentum operator will coincide with those related to the real momentum of the particle. For example, consider a stationary wave, i.e. a superposition of plane waves going in opposite directions:

$$
\psi(x, t)=e^{+\frac{i}{\hbar} p x}+e^{-\frac{i}{\hbar} p x}
$$

For this state, the eigenvalue of the kinetic energy operator will be different from the real kinetic energy of the particle. In fact, the eigenvalue of the kinetic energy operator $\hat{E}_{k}=\frac{\hat{P}}{2 m}$ takes the constant value:

$$
E_{k}=\frac{p}{2 m} \neq 0
$$

whereas the real kinetic energy $E_{k}^{r e a l}$ is zero, since the actual velocity of the particle is zero (for a stationary wave, the particle remains at rest in a non-nodal point):

$$
\nabla S=p-p=0 \longrightarrow E_{k}^{r e a l}=\frac{(\nabla S)^{2}}{2 m}=0
$$

In conclusion: even if the momentum eigenvalue (eigenvalue of the momentum operator $\hat{P}_{x}=$ $\left.-i \hbar \nabla_{x}\right)$ and the real momentum of the particle $(p=\nabla S)$ are a priori different concepts, still they are not completely disentangled. In fact, both the momentum operator and the real momentum of the particle are mathematically expressed by a derivative of the wave function in the position variable. For this reason, when we multiply a (position-basis) wave function by a relative phase, this will generally affect the value of both the real momentum of the particle (computed via the gradient of the phase) and the momentum eigenvalue (computed via the gradient of the wave function). The new real particle momentum will thus be linked to the new momentum eigenvalue and, consequently, to the new momentum probability distribution. For simple cases, such as in the case of plane waves illustrated above, the momentum eigenvalue and the real particle momentum will coincide. For (at least) such simple cases, the variation of the actual velocity of the particles induced by the relative phase provides an intuitive physical explanation to the change of the momentum probability distribution.

\subsection{Statistical interpretation and individual description}

The quantum continuity equation-when realistically interpreted--describes a collection of particles $|\psi|^{2}$-distributed and moving with velocity $v=\frac{\nabla S}{m}$. However-one may ask-why should we interpret such equation in realistic terms? To answer this question, consider the following set of arguments:

1. Physical $|\psi|^{2}$. In standard quantum mechanics, what is assigned a physical meaning is not the wave function $\psi$ per se, but the absolute square of the wave function $|\psi|^{2}$, interpreted as particle probability density. The integral of $|\psi|^{2}$ is thus the probability of finding the particle in a given region if a position measurement is performed on that region.

2. Physical $\frac{d|\psi|^{2}}{d t}$. If $|\psi|^{2}$ has a physical meaning, then it is natural to think of the dynamical equation associated to this quantity as having a physical meaning as well. This equation (the time evolution of $|\psi|^{2}$ ) is the quantum continuity equation: $\frac{\partial|\psi|^{2}}{\partial t}+\nabla \cdot J=0$. 
3. Analogy with the classical continuity equation. Based on step (2), the analogy between classical and quantum continuity equation should be best regarded as a physical analogy and not just a mathematical formal analogy.

The three steps considered above, even if not conclusive, seem nevertheless to suggest a realistic interpretation of the quantum continuity equation. It follows that a physical interpretation of the terms included in that equation, such as the particle probability density $|\psi|^{2}$ and the velocity term $v=\nabla S / m$ are not only in agreement but naturally suggested by (a fairly realistic reading of) standard quantum mechanics. With this, I do not intend that a particle ontology is the only interpretation available here, but that, if we take a realistic stance of the quantum continuity equation (for the reasons stated above), then a particle ontology follows as the most natural interpretation for the velocity term derived from that equation. Furthermore, we have noted (Section 3) that, contrary to Sakurai's claim, a real particle velocity is not in contradiction with the position-momentum uncertainty principle, for the latter is best regarded as a statistical principle that applies to ensembles of systems and not to single systems. Quantum mechanics, in its standard interpretation, is indeed a statistical theory: the physical content of the theory is primary given by measurement results (eigenvalues of the measured observables), collectedafter many measurements performed on initially prepared systems-into empirical distributions. Such distributions can be finally used to describe the relevant physical properties of the system, such as the mean value and the standard deviation of the measured observables.

Nevertheless, such statistical interpretation of quantum mechanics cannot be regarded as the fundamental description: statistics and empirical distributions are always collected from and computed after sequences of single events, i.e. events generated by individual systems. Quantum mechanics, when realistically interpreted, is nevertheless able to provide such a dynamical description of individual systems in terms of particles having well-defined positions and velocities. This interpretation corresponds to the most known "hidden variable" theory, i.e. the de Broglie-Bohm theory. ${ }^{11}$ It is important to note, however, that the hidden variable $x$ is not artificially added to but mathematically derived from the quantum formalism: the position variable is already present in the standard definition of the probability density $|\psi(x, t)|^{2}$, and the velocity variable in the quantum flux term $\left(J=\rho v=|\psi|^{2} \frac{\nabla S}{m}\right)$ of the quantum continuity equation, mathematically derived from the Schrödinger equation.

That a statistical interpretation must reduce, on a deeper level, to a theory about individual systems was pointed out by Einstein $(1953)^{12}$ commenting on the Born's statistical interpretation of the wave function:

[...]the acceptable interpretation of the Schrödinger equation is the statistical interpretation given by Born. However this does not provide any real description of an

\footnotetext{
${ }^{11}$ According to the standard definition (see e.g. Harrigan and Spekkens (2010)), the de Broglie-Bohm theory is a hidden variable theory for the complete ontological state of the system is represented by $\lambda=(\psi, x)$, that is, the wave function of the system is supplemented with the variable $x$. The variable $\mathrm{x}$ takes the name of "hidden variable" since, even when we have perfect knowledge of the wave function (for example, when the wave function is a pure state), we still do not know the exact positions of the particles, but only that they are statistically distributed according to $|\psi(x)|^{2}$.

${ }^{12}$ In this essay, Einstein makes an objection to Bohm's theory, claiming that it fails to describe the correct classical limit for a massive point-particle in an infinite potential well. He shows, in fact, that the particle remains at rest in a non-nodal point inside the well even for large mass and small de Broglie wave-length (high energy), when the classical regime is expected to arise. On this basis, he excludes Bohm's theory as a valid option. However, the example made by Einstein does not take into account the interaction with the environment and, consequently, decoherence effects, which is nowadays the standard framework for the classical limit (historically, indeed, decoherence theory will be developed only around 1980s). When decoherence effects are taken into account, Bohm's theory describes the correct classical regime, with the particle inside the well moving according to Newton's theory (see e.g. (Bohm and Hiley 1993, Ch. 8)).
} 
individual system, but only statistical predictions about ensembles of systems.

In my opinion it is in principle not satisfactory that such a theoretical framework should lie at the foundations of physics, especially since the objective describability of an individual macroscopic system (description of the "real state") cannot be given up without the picture of the physical world dissolving in a cloud, as it were. Finally, the judgement that physics must strive towards a real description of an individual system, is unavoidable. Nature as a whole can only thought of as an individual (singly existing) system and not as an "ensemble of systems".

To my view, one candidate to recover such individual description of Nature is given by the de Broglie-Bohm theory. This theory explains the usual quantum statistics in terms of "singlyexisting" individual systems, i.e. particles $|\psi|^{2}$-distributed and moving with velocity $v=$ $\nabla S / m$. Such particle dynamics, however, is not artificially added to but mathematically extracted from the basic dynamical equations of standard quantum mechanics.

\section{Conclusions}

I have shown that quantum mechanics may be naturally interpreted as a theory of particles in motion, with position probability distribution $\rho=|\psi|^{2}$ and velocity $v=\nabla S / m$. These pieces of information are not added to the standard formalism of quantum mechanics, but extracted from the quantum continuity equation, specifically from the quantum flux $J=\rho v$. Despite the apparent analogy with the classical flux, the velocity component of the flux is generally not interpreted as a proper velocity, for this is thought to imply a violation of the positionmomentum uncertainty principle. However, on a careful analysis, there is no tension between the two: the uncertainty principle refers to the statistics of measurements on ensemble of systems and not to simultaneous position and momentum measurements on single systems. As such, it does not prevent systems from having well-defined values of position and momentum. The quantum flux then naturally describes, in analogy with its classical analogue, a collection of particles $|\psi|^{2}$-distributed and moving with velocity $v=\nabla S / m$. This quantum description corresponds to the most known "hidden-variable" theory, i.e. the de Broglie-Bohm theory, which describes the quantum measurement statistics in terms of "singly existing" individual systems. Finally, I have shown that, using this framework, it is possible to provide (at least, for simple cases) an intuitive explanation for the change of the momentum probability distribution that occurs when the wave function of a system is multiplied by a relative phase. 


\section{References}

Ballentine, L. E. (1970). The Statistical Interpretaion of Quantum Mechanics. Reviews of Modern Physics, 42(4):358-381.

Ballentine, L. E. (1998). Quantum Mechanics. A Modern Development. Wolrd Scientific.

Bohm, D. and Hiley, B. (1993). The Undivided Universe: An Ontological Interpretation of Quantum Theory. Routledge.

Bowman, G. E. (2008). Essential quantum mechanics. Oxford University Press.

Einstein, A. (1953). Elementare Überlegungen zur Interpretation der Grundlagen der Quantenmechanik; English Translation: Elementary Considerations on the Interpretation of the Foundations of Quantum Mechanics (arXiv:1107.3701v1 [physics.hist-ph]). In Scientific Papers Presented to Max Born on His Retirement from the Tait Chair of Natural Philosophy in the University of Edinburgh, pages 33-40. Hafner Publishing.

Harrigan, N. and Spekkens, R. W. (2010). Einstein, incompleteness and the epistemic view of quantum states. Foundations of Physics, 4:125-157.

Hilgevoord, J. and Uffink, J. (2016). The Uncertainty Principle. Stanford Encyclopedia of Philosophy.

Holland, P. (1993). The Quantum Theory of Motion. An Account of the de Broglie-Bohm Causal Interpretation of Quantum Mechanics. Cambridge University Press.

Jauch, W. (1993). Heisenberg's uncertainty relation and thermal vibrations in crystals. American Journal of Physics, 61(10):929-932.

Kennard, E. H. (1927). Zur Quantenmechanik einfacher Bewegungstypen. Zeitschrift für Physik, 44:326-352.

Margenau, H. (1963). Measurements in quantum mechanics. Annals of Physics, 23(469-485).

Robertson, H. P. (1929). The Uncertainty Principle. Physical Review, 34(1):163-164.

Sakurai, J. J. (1994). Modern Quantum Mechanics. Addison-Wesley Publishing Company.

Schlosshauer, M. A. (2007). Decoherence and the Quantum-to-Classical Transition. Springer. 